\title{
Initial Flux Decline and Initial Rejection Increase for Swollen Ionic Membranes
}

\author{
P. M. VAN DER VELDEN and C. A. SMOLDERS, Twente University of \\ Technology, Enschede, The Netherlands
}

\begin{abstract}
Synopsis
During the initial stage of operation, membrane performance in hyperfiltration experiments will be time dependent. For swollen ionic membranes, fluxes show an initial decrease while rejections initially increase in magnitude. These phenomena, also referred to as compaction, can be described by a model proposed here in which the wet membrane thickness decreases in time as a combined response to stepwise changed process parameters pressure and concentration. The response has been described by a linear first-order differential equation and worked out by use of known concepts of flux and rejection for swollen ionic membranes. Experimental data appear to be in good agreement with model predictions.
\end{abstract}

\section{INTRODUCTION}

Compaction is a concept in membrane research generally used to indicate phenomena like initial flux decline. Nonlinear relations between rejection or flux on the one hand and the operating pressure on the other are also ascribed to "compaction." Because of the practical importance of these phenomena, several authors studied flux changes in time. Bert ${ }^{1}$ found for cellulose acetate membranes that the permeability is directly associated with the state of hydration for a given membrane, the hydration being determined by the pressure applied. Yasuda and Schindler ${ }^{2,3}$ gave a mathematical description for the relation between the state of hydration and the flux for highly hydrated membranes. The influence of membrane thickness $d$ on the permeability through cellulose acetate membranes was studied by Baayers and Rosen ${ }^{4}$; they found a linear relationship between the waterflux through the membrane and the inverse value of $d$.

The influence of the applied pressure on the flux has also been investigated. For ionic membranes, Johnson ${ }^{5}$ and Lopatin and Newey ${ }^{6}$ found that flux and rejection increased nonlinearly with increasing pressure. The latter authors also studied several ionic membranes for the effect of different concentrations of $\mathrm{NaCl}$ solution on membrane swelling values and for the effect of pressure on membrane deswelling. The compression under pressure followed closely the theory of Katchalski, Lifson, and Eisenberg. ${ }^{7}$ The swelling values in $\mathrm{NaCl}$ solutions were clearly dependent on the salt concentration: differences of up to $50 \%$ in water content of the membranes were found at normal pressures for solutions between zero and $0.1 \mathrm{~N} \mathrm{NaCl}$. 
The objective of our investigation was to study the initial flux decline and initial rejection increase for swollen ionic membranes. Although all references cited above deal more or less with the compaction phenomenon, no model that could explain the simultaneous occurrence of an initial flux decline and an initial rejection increase in hyperfiltration experiments has been given up until now. In this paper such a model is proposed.

\section{MODEL}

The exact amount of membrane water in a membrane under operation can be determined at any moment with great difficulty only, since the hydration $H$ is a function of pressure $p$ and salt concentration $c$. We will describe the flux and rejection in terms of the wet membrane thickness $d_{w}$ as a variable. It is known from process dynamics that the responses on simultaneous step changes in $p$ and $c$ can be summed. This is of importance here, since the decline of the swollen membrane thickness $d_{w}$ in time cannot be ascribed to the influence of either pressure $p$ or concentration $c$ alone. We therefore propose a model in which the wet membrane thickness decreases in time as a combined response to the stepwise changed process parameters pressure and concentration.

In an ideal relaxation process, the rate of decrease of $d_{w}$ is dependent on the deviation of $d_{w}$ from the equilibrium value $d_{e q}$. This relaxation process can be described by a linear first-order differential equation, with the solution

$$
d_{w}-d_{e q}=\left(d_{w}^{0}-d_{e q}\right) \exp (-t / \tau)
$$

Experimentally, eq. (1) cannot be verified directly. Since flux and rejection are functions of the volume fraction of hydration water in the membrane and wet membrane thickness $d_{w}$, flux and rejection can be studied as functions of time.

To describe the initial changes in flux and rejection, we write the differential with respect to $t$ as follows:

$$
\frac{d X}{d t}=\frac{\partial X}{\partial d_{w}} \cdot \frac{\partial d_{w}}{\partial t}
$$

with $X=-J_{w}$ or $X=R$. If $\partial X / \partial d_{w}$ is a constant (which assumption will be discussed below), we can represent the initial flux decline and initial rejection increase by

$$
\begin{gathered}
\ln \left\{-\frac{d J_{w}}{d t}\right\}_{t}=K_{1}-\frac{t}{\tau} \\
\ln \left\{\frac{d R}{d t}\right\}_{t}=K_{2}-\frac{t}{\tau}
\end{gathered}
$$

These equations have been tested experimentally, and results will be discussed in the results section. But first we will explore from known concepts of flux and rejection for swollen ionic membranes which conditions should be fulfilled for eqs. (3a) and (3b) to be valid. 


\section{Initial Flux Decline}

Yasuda and Schindler,3 used the following equation to describe the influence of the volume fraction of hydration water in the membrane on the water flux for highly swollen membranes:

$$
J_{w}=K_{0}(\Delta p-\Delta \pi) \cdot \exp \left[-B\left(\frac{1-H}{H}\right)\right] .
$$

In the case of flat homogeneous (nonporous) membranes, the following relation between $H$ and the membrane thickness is valid:

$$
H=\frac{d_{w}-d_{d}}{d_{w}} .
$$

Combination of eqs (1), (2), (4), and (5) gives

$$
\frac{d J_{w}}{d t}=-K \cdot A \cdot \exp (-t / \tau)
$$

with

$$
\begin{aligned}
K & =\left[K_{0}(\Delta p-\Delta \pi) \cdot B \cdot d_{d} \cdot\left\{\left(d_{w}^{0}-d_{e q}\right) / \tau\right\}\right] \\
A & =\left\{\left(d_{w}-d_{d}\right)^{2} \cdot \exp \left\{B \cdot d_{d} /\left(d_{w}-d_{d}\right)\right\}\right\}^{-1} .
\end{aligned}
$$

The term $\left(d_{w}-d_{d}\right)$ varies from $\left(d_{w}^{0}-d_{d}\right)$ to $\left(d_{e q}-d_{d}\right)$ and can be expressed by

$$
\left(d_{w}-d_{d}\right)=\omega \cdot d_{d}
$$

with

$$
\frac{d_{e q}}{d_{d}} \leq \omega+1 \leq \frac{d_{w}^{0}}{d_{d}} .
$$

With this expression, eq. (6) can be rewritten as

$$
\ln \left(-\frac{d J_{w}}{d t}\right)=K_{1}-\frac{t}{\tau}-\ln \left[\omega^{2} \cdot \exp (B / \omega)\right]
$$

with

$$
K_{1}=\ln \left\{K_{0}(\Delta p-\Delta \pi) B\left(d_{w}^{0}-d_{e q}\right) / d_{d} \tau\right\} .
$$

Since $\omega$ depends on $t$, the term $\left(K_{1}-\ln \left[\omega^{2} \cdot \exp (B / \omega)\right]\right)$ can decrease as $d_{w}$ approaches the equilibrium wet membrane thickness $d_{e q}$.

Comparing eq (8) with eq. (3a), we expect that eq. (3a) will be followed for initial flux decline if the term $\ln \left[\omega^{2} \cdot \exp (B / \omega)\right]$ does not depend too much on $\mathrm{t}$ in comparison with the term $t / \tau$.

In the description given above, the possible influence of changes in $\Delta \pi$ on the flux, eq. (4), through an initial increase in the rejection has been neglected.

\section{Initial Rejection Increase}

According to Shor et al., ${ }^{8}$ the rejection of an ionic membrane can be written as 


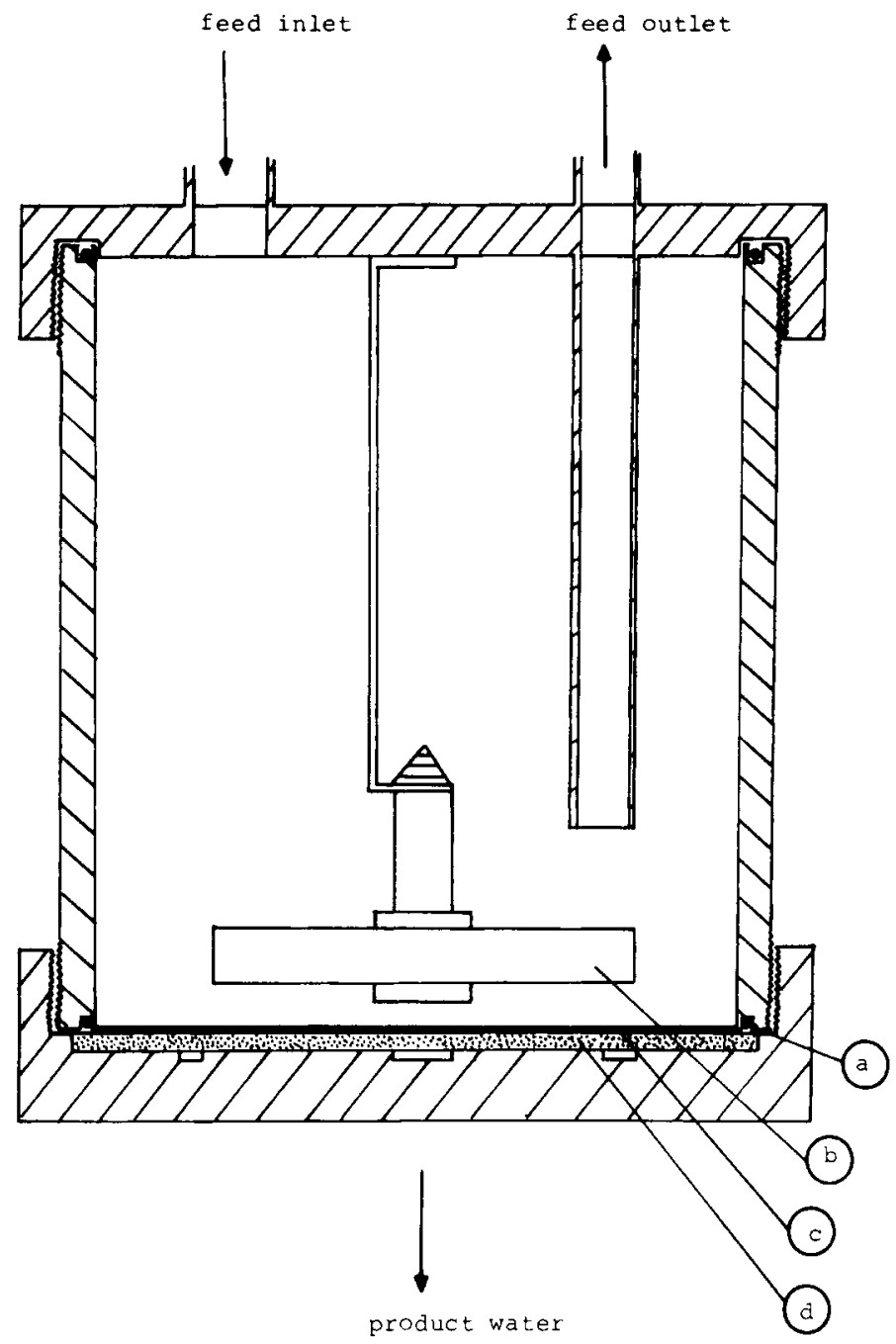

Fig. 1. High-pressure reverse osmosis cell: (a) O-ring; (b) magnetic stirrer; (c) membrane; (d) sintered metal disc.

$$
\mathrm{D}_{\alpha}^{*}\left[\frac{C^{*}}{c_{s}}+z \mathrm{D}_{c x}^{*}\right]^{z} \Gamma^{z+1}=z^{z}
$$

where $R=\left(1-\beta D_{\alpha}^{*}\right)$ and generally $\beta=1$. After substitution of $\left\{C_{d^{*}}^{*}(1-\right.$ $H / H)\}$ for $C^{*}$ and eq. (5) for $H$, eq. (9) can be differentiated with respect to $d_{w}$. Assuming $d \Gamma / d d_{w}=0$, eq. (2) can be worked out to give $\ln \left(\frac{d R}{d t}\right)_{t}=\left[\ln \left\{\frac{C_{d}^{*}\left(d_{w}^{0}-d_{e q}\right)}{c_{s} \cdot \tau \cdot d_{d}}\right\}\right]-\frac{t}{\tau}$

$$
+\ln \left\{\frac{z(1-R)}{\omega^{2}(1-R)\left(z+z^{2}\right)+\left(\frac{C_{d}^{*} \cdot \omega}{c_{s}}\right)}\right\} .
$$

Since $\omega^{2}(1-R)\left(z+z^{2}\right)$ is much smaller than $\left(\omega \cdot C_{d}^{*} / c_{s}\right)$, eq. (10) becomes 


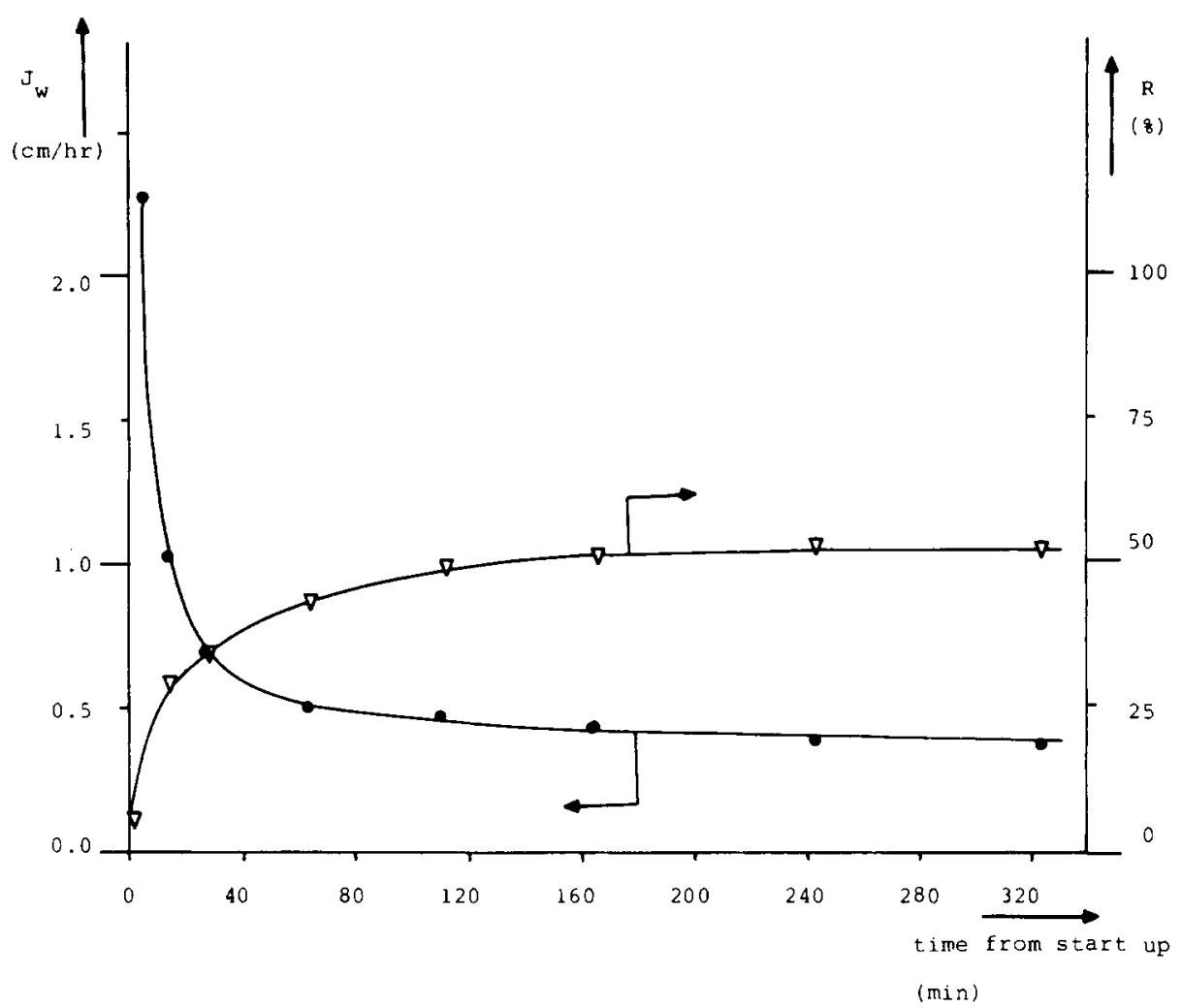

Fig. 2. Initial flux decline and initial rejection increase for an ionic S-I-S-48.3 membrane at 40 atm with a $2100 \mathrm{ppm} \mathrm{NaCl}$ feed solution.

$$
\ln \left(\frac{d R}{d t}\right)=\ln \left[\frac{\left(d_{w}^{0}-d_{e q}\right) \cdot z \cdot(1-R)}{\omega \cdot \tau \cdot d_{d}}\right]-\frac{t}{\tau} .
$$

We see that eqs. (11) and (3b) become identical if $\ln (1-R) / \omega$ does not depend on $t$. With the help of eqs. (1), (7), and (11), eq. (12) can be shown to be valid for $z=1$ (and in general for all $n-n$ electrolytes):

$$
\frac{\partial \ln \left[\frac{1-R}{\omega}\right]}{\partial t}=-\left\{\frac{1}{\omega} \cdot \frac{d \omega}{d t}+\frac{1}{1-R} \cdot \frac{d R}{d t}\right\}=0
$$

\section{EXPERIMENTAL}

\section{Membranes}

The negatively charged polymeric material used for preparing the membranes was made from a commercially available polystyrene-polyisoprenepolystyrene (S-I-S) block copolymer (Cariflex TR 1108). The preparative methods have been described elsewhere ${ }^{6,9}$ and are summarized below.

S-I-S-48.3. To a $8 \% \mathrm{~S}-\mathrm{I}-\mathrm{S}$ solution in dioxane, $\mathrm{HSO}_{3} \mathrm{Cl}$ was added at 
room temperature. The polyisoprene middle block reacts with the acid as follows ${ }^{6}$ :

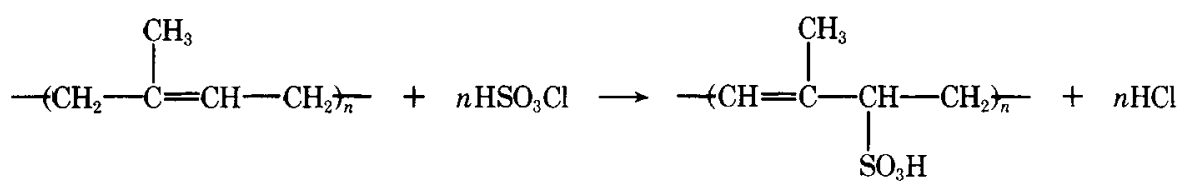

If all the isoprene would be modified in this manner, we would obtain a water soluble polyelectrolyte. For membrane preparation purposes we modified part of the isoprene in the middle block (48.3\%). The polymer solution was cast on a glass plate with a doctor's knife, the solvent evaporated overnight, and finally the film was immersed in a $\mathrm{KOH}$ solution to form the salt.

S-I-S-S-20-HOM. To a $12.8 \%$ S-I-S solution in toluene, a certain amount of $\mathrm{N}$-chlorosulfonyl isocyanate (NCSI) was added. The NCSI reacts with isoprene according to

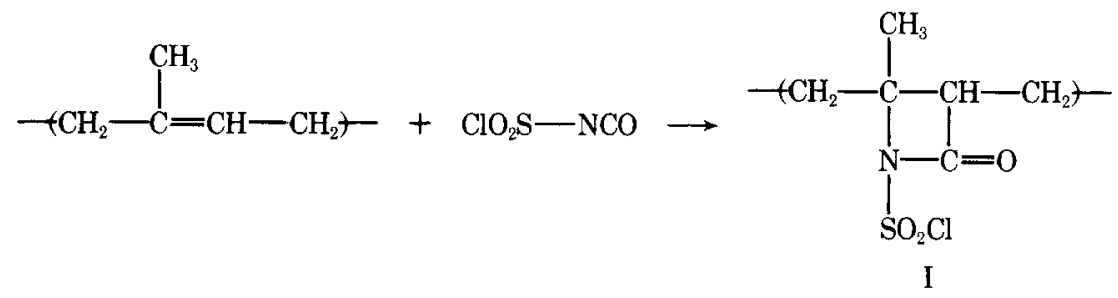

The ratio (moles NCSI added/moles isoprene) was 20\%. From a solution of polymer $I$ in toluene a film was cast on a glass plate. After evaporation of the toluene, the film was hydrolysed in $1.65 \mathrm{M}$ ammonia for $45 \mathrm{~min}$. Polymer I reacts with ammonia as follows ${ }^{9}$ :<smiles>[R]C1([R])C(=O)N(S(=O)(=O)[O-])C1([R])[R]</smiles>

All membranes were rinsed with water before they were used. During the test, the membranes were supported by Schleicher and Schuell filter paper.

\section{Apparatus}

An Amicon high-pressure cell type 420 (Fig. 1) was used for the hyperfiltration tests. The cell was connected with a salt solution reservoir and the whole system was put under pressure by means of nitrogen gas. The salt solution directly above the membrane was mixed with a magnetic stirrer (450 cycles per $\mathrm{min}$ ). The rejection was determined by measuring the salt concentration conductometrically:

$$
R=\frac{c_{\text {feed }}-c_{\text {permeate }}}{c_{\text {feed }}} \cdot 100 \%
$$




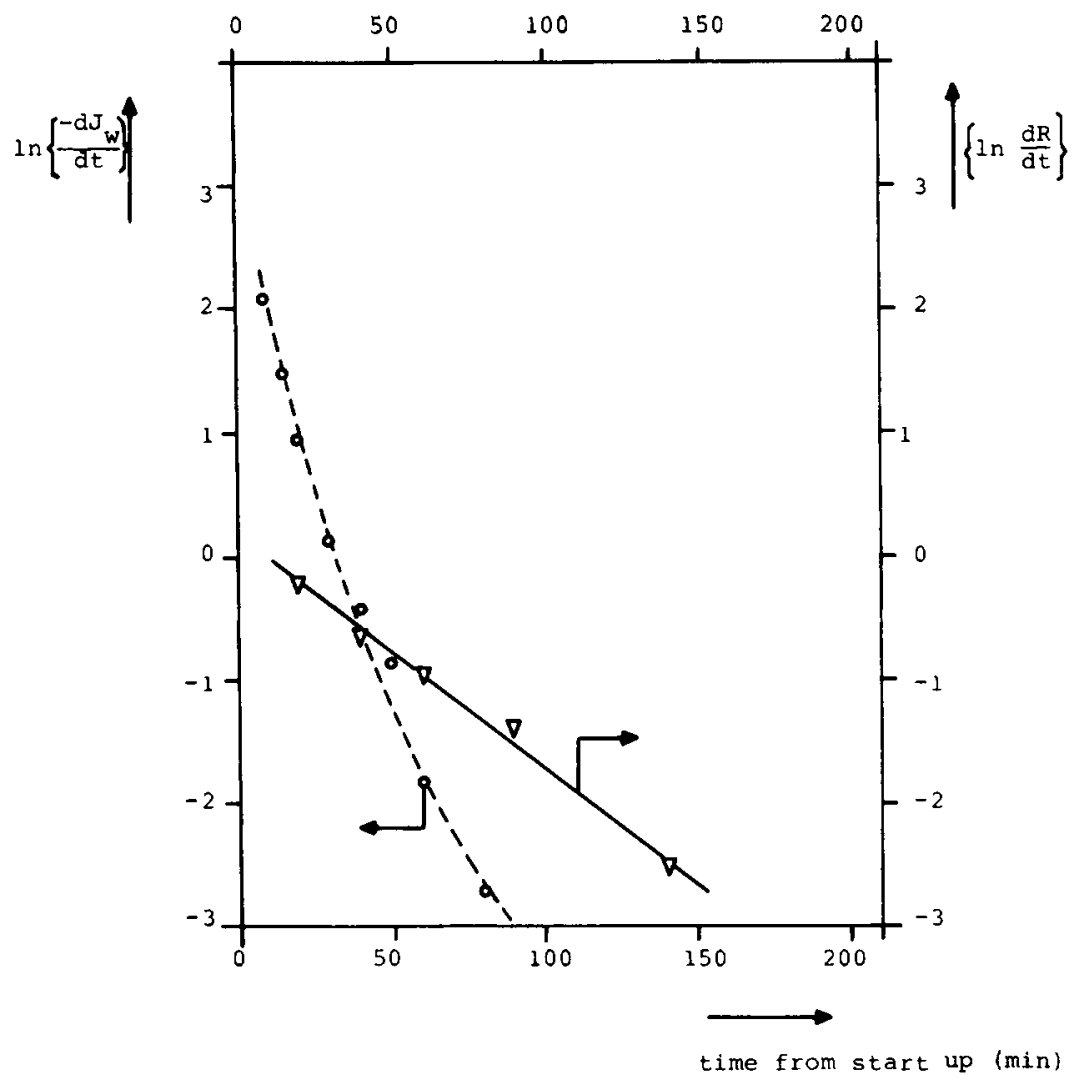

Fig. 3. Plot of the natural logarithm of $d R / d t$ and $-d J_{\omega} / d t$ vs. time for an ionic S-I-S-48.3 membrane at $40 \mathrm{~atm}$, with a $2100 \mathrm{ppm} \mathrm{NaCl}$ feed solution.

\section{RESULTS AND DISCUSSION}

Equations (3a) and (3b) were tested with two different types of ionic membranes. The sulfonated S-I-S-48.3 membrane was tested with a 2100 $\mathrm{ppm} \mathrm{NaCl}$ solution at $40 \mathrm{~atm}$. The initial flux decline and rejection increase are shown in Figure 2. In Figure $3, \ln (d R / d t)$ and $\ln \left(-d J_{w} / d t\right)$ are given as a function of time.

The S-I-S-S-20-HOM membranes were tested with different salts and pressures. In Figures $4 \mathrm{a}$ and $4 \mathrm{~b}$, the results are given for tests carried out with a $4500 \mathrm{ppm} \mathrm{NaCl}$ solution at $40 \mathrm{~atm}$. For this membrane, we could not measure a significant initial flux decline. In Figure 5a, the rejections are shown for tests at 30 and $40 \mathrm{~atm}$, respectively, with a $7700 \mathrm{ppm} \mathrm{Na}_{2} \mathrm{SO}_{4}$ solution. The final rejection values at these two pressures are almost equal, indicating that $d_{e q}(30 \mathrm{~atm})$ is just a little larger than $d_{e q}(40 \mathrm{~atm})$.

Since $\mathrm{Na}_{2} \mathrm{SO}_{4}$ is not a $\mathrm{n}-\mathrm{n}$ electrolyte, $\partial \ln [(1-R) / \omega] / \partial t$ in eq. (12) becomes negative, while $K_{2}$ in eq. (3b) decreases in time according to

$$
K_{2}=\ln \left[\frac{z\left(d_{w}^{0}-d_{e q}\right)}{\tau d_{d}}\right]+\ln \left(\frac{1-R}{\omega}\right) .
$$

Since now $K_{2}$ is time dependent, the linear relation between $\ln (d R / d t)$ and $t$ does not hold any longer. In Figure $5 b$, we can see that for both experiments 


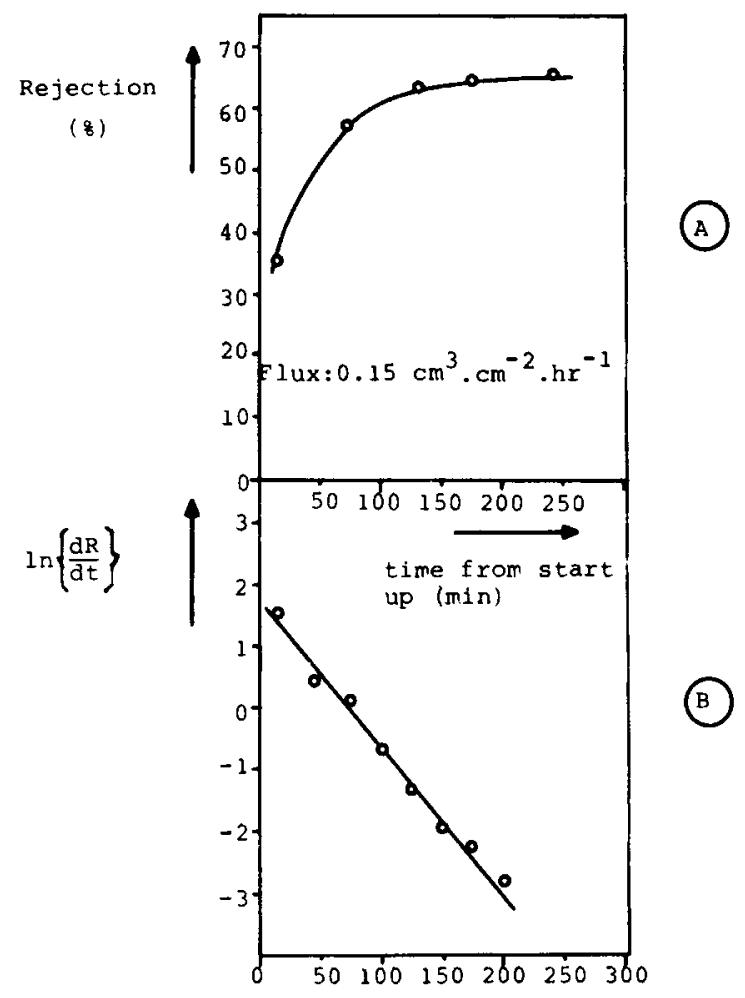

Fig. 4. Initial rejection increase for a S-I-S-S-20-HOM membrane at 40 atm, with a $4500 \mathrm{ppm}$ $\mathrm{NaCl}$ feed solution.

the experimental points deviate more and more from the drawn lines with increasing time. Calculation of a relaxation time from Figure $5 \mathrm{~b}$ is not possible for this reason.

Figure 3 demonstrates that the slopes for the lines describing the initial flux decline and rejection increase differ from each other. According to eqs. ( $3 a$.) and ( $3 b)$, however, both slopes should be equal to $1 / \tau$. The deviation between experimental data and model predictions may be due to the presence of the term $\ln \left[\omega^{2} \cdot \exp (B / \omega)\right]$ in eq. (8). The experimental flux data in Figure 3 indicate that the term $\ln \left[\omega^{2} \cdot \exp (B / \omega)\right]$ is not constant in time. Consequently, a relaxation time cannot be calculated from our experimental flux data.

A similar phenomenon can be observed in the work of Yasuda and Lamaze. ${ }^{10,11}$ These authors investigated the dependence of the permeability of solutes through uncharged membranes, $P_{2,13}$, when hydration $H$ varied. They found the following relation between $\ln P_{2,13}$ and $1 / H$ :

$$
\ln \left(P_{2,13} / D_{2,1}\right)=\ln \left[\alpha \cdot H \cdot \phi\left(q_{2}\right)\right]-B\left(q_{2} / V_{f, 1}\right)\left[\frac{1}{H}-1\right]
$$

Yasuda and Lamaze explained their experimental data by a linear relation between $\ln P_{2,13}$ and $1 / H$, without exploring the significance of the term $\ln \mathrm{H}$.

In Figure 6, the model is checked with data for hydrous $\mathrm{Zr}$ (IV) oxide membranes. ${ }^{5}$ Both $\ln (d R / d t)$ and $\ln \left(-d J_{w} / d t\right)$ are linear in $t$ with a negative 


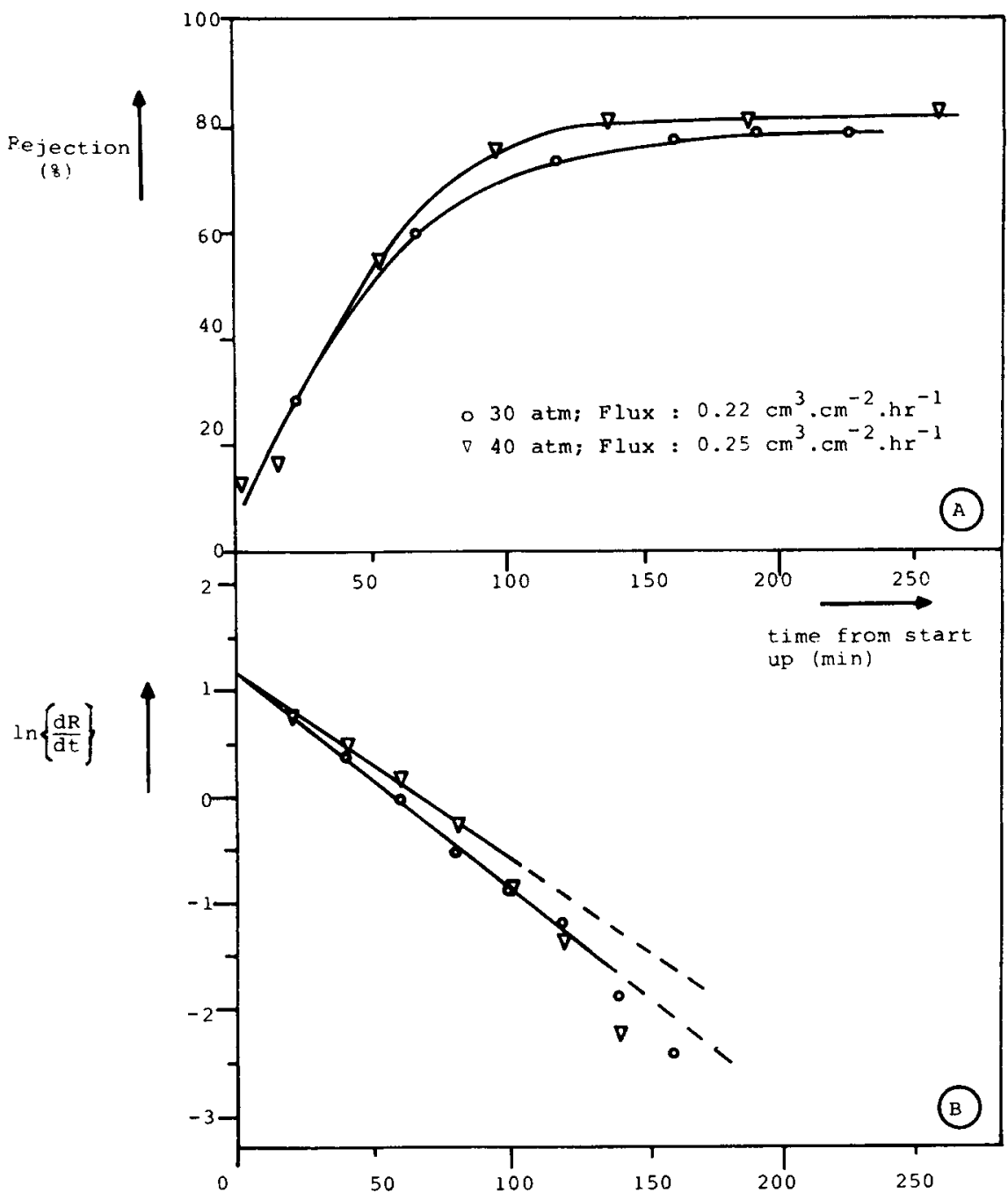

Fig. 5. Initial rejection increase for a S-I-S-S-20-HOM membrane at $30 \mathrm{~atm}(\mathrm{O})$ and $40 \mathrm{~atm}$ $(\nabla)$, with a $7700 \mathrm{ppm} \mathrm{Na}_{2} \mathrm{SO}_{4}$ feed solution.

slope which is practically equal for both lines, showing that eqs. (3a) and (3b) are followed. In the foregoing discussion, the time dependence of $H$ was studied for swollen ionic membranes. This initial deswelling of the membrane is due to removal of the loosely bound hydration water.

Yasuda and Schindler ${ }^{2}$ approached the flux also in a different way than given in eq. (4), by using a relation in which the flux is described by a diffusion dependent constant $K_{d}$ and a viscous flow dependent constant $K_{f}$ :

$$
J_{w}=\left(K_{d}+K_{f}\right)(\Delta p-\Delta \pi)
$$

According to Peterlin, Yasuda, and Olf ${ }^{12}$ the viscous flow-dependent term $K_{f}$ is linear in $H /(1-H)=\omega$. This means that $K_{f}$ as well as $d_{w}$ decrease exponentially in time, as a response to the stepwise changed process parameters.

Since the initial decline of $d_{w}$ at constant applied pressure can be compared with the decrease of $d_{e q}$ when the applied pressure increases, the analy- 


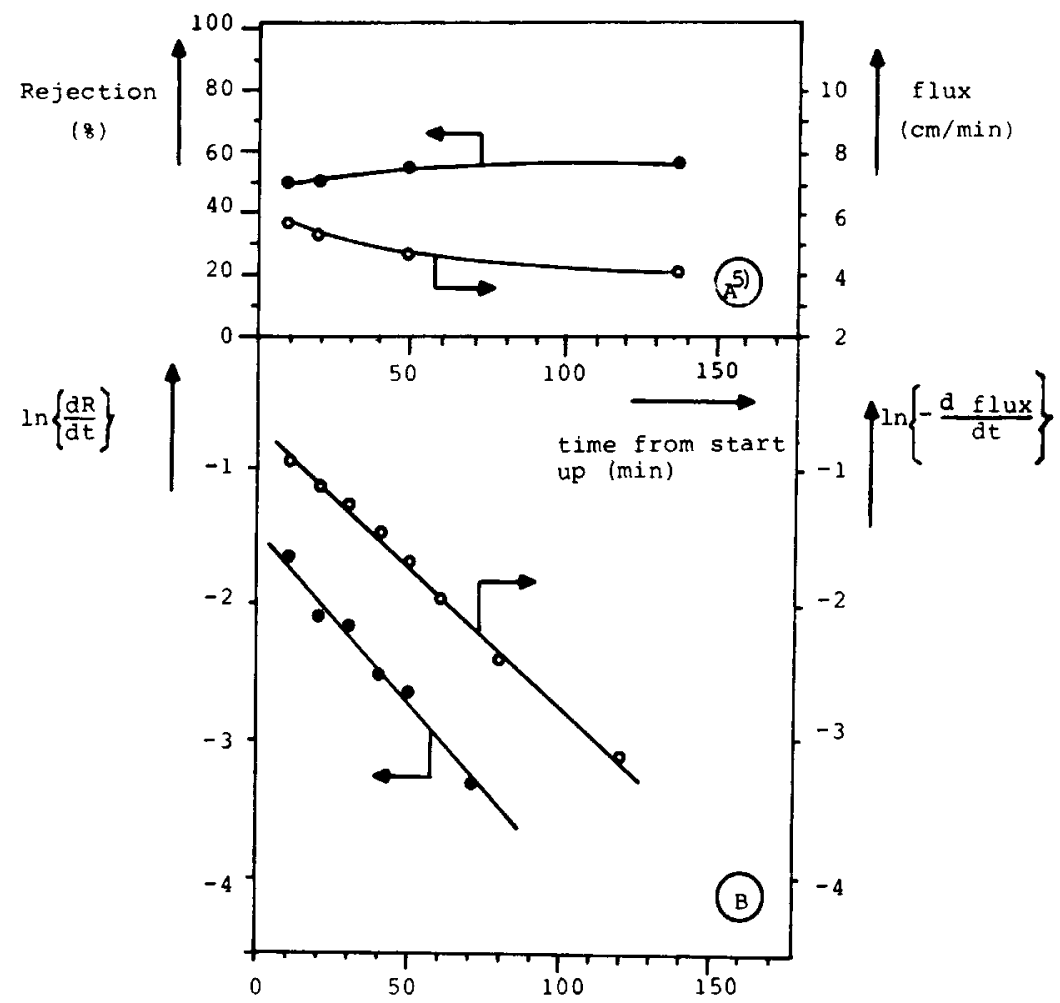

Fig. 6. Initial flux decline and rejection increase for hydrous $\mathrm{Zr}$ (IV) oxide membranes at 67 atm; feed solution contains $2900 \mathrm{ppm} \mathrm{NaCl}$ and $10^{-4} \mathrm{M}$ hydrous $\mathrm{Zr}$ (IV) oxide. ${ }^{5}$

sis given here can also be applied to flux/rejection measurements at different pressures.

\section{CONCLUSIONS}

The physical meaning of the model proposed here lies in the initial removal of the loosely bound hydration water due to the influence of pressure as well as salt concentration. This removal of water can be described by an exponential decrease of the wet membrane thickness $d_{w}$.

The validity of the model is demonstrated for rejection measurements with 1:1 electrolytes and contains a function to explain the deviation of asymmetrical electrolytes. Generally, it is not possible to calculate relaxation times from the flux data due to uncertainty in the value of $\omega$, assumed constant in time.

Experimental data show that information on membrane performance can be obtained by dynamic measurements.

\section{Greek Symbols}

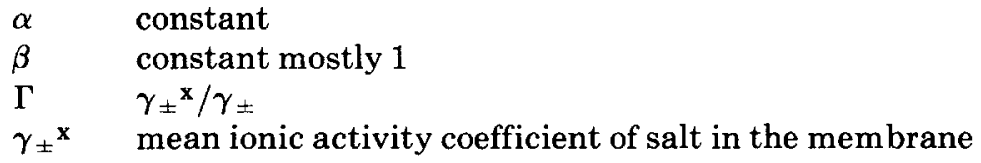


$\gamma_{ \pm} \quad$ mean ionic activity coefficient of salt in the salt solution

$\phi\left(q_{2}\right)$ this term describes the sieve mechanism by which small molecules are permitted to diffuse and larger molecules are rejected because the macromolecular network has no hole of appropriate size

$\Delta \pi \quad$ osmotic pressure difference across the membrane (atm)

$\tau \quad$ relaxation time ( $\mathrm{min}$ )

$\omega \quad$ time-dependent variable

\section{Nomenclature}

\section{$B, C \quad$ constants}

$c_{f} \quad$ salt concentration in filtrate (mole/l.)

$c_{m} \quad$ salt concentration in membrane (mole/l.)

$c_{s} \quad$ salt concentration in salt solution (mole/l.)

$C^{*} \quad$ membrane capacity (eq/ $\mathrm{kg}$ membrane water)

$C_{d} * \quad$ ion exchange capacity (meq/g dry polymer)

$d_{e q} \quad$ equilibrium wet membrane thickness at $t=\infty$ under operating pressure $(\mathrm{cm}$

$d_{d} \quad$ dry membrane thickness at $t=\infty$ under operating pressure $(\mathrm{cm})$

$d_{w} \quad$ wet membrane thickness at time $t$ and under operating pressure $(\mathrm{cm})$

$d_{w}^{0} \quad$ wet membrane thickness at zero time $(\mathrm{cm})$

$D_{\alpha}{ }^{*} \quad$ equilibrium distribution coefficient of component $\alpha\left(c_{m} / c_{s}\right)$

$D_{2,1}$ diffusion coefficient of solute in water $\left(\mathrm{cm}^{2} / \mathrm{sec}\right)$

$H \quad$ volume fraction of water in the membrane

$J_{w} \quad$ water flux through the membrane $\left(\mathrm{cm}^{3} / \mathrm{cm}^{2} \mathrm{hr}\right)$

$K_{i} \quad$ constants

$\Delta p \quad$ applied pressure (atm)

$P_{2,13}$ permeability of solute through water-swollen membrane $\left(\mathrm{cm}^{2} / \mathrm{sec}\right)$

$q_{2} \quad$ cross-sectional area of the diffusing molecule

$R \quad$ rejection $\left(1-\frac{c_{f}}{c_{s}}\right)$

$t \quad$ time from startup ( $\mathrm{min}$ )

$V_{f, 1} \quad$ free volume of pure water

$X \quad R$ or $-J_{w}$

$z \quad$ charge of coion

\section{References}

1. J. L. Bert, I. Fatt, and D. N. Saraf, Appl. Polym. Symp., 13, 105 (1973).

2. H. Yasuda and A. Schindler, in Reverse Osmosis Membrane Research, H. K. Lonsdale and H. E. Podall, eds., Plenum Press, New York, 1972, pp. 299-316.

3. H. Yasuda, C. E. Lamaze, and A. Schindler, J. Polym. Sci. A-2, 9, 1579 (1971).

4. L. Baayers and S. L. Rosen, J. Appl. Polym. Sci., 16, 663 (1972).

5. J. S. Johnson, Jr. Polym. Prepr., 12, 436 (1971).

6. G. Lopatin and H. A. Newey, Office of Saline Water, R \& D Report No. 690, U.S. Dept. of the Interior, May 1971.

7. A. Katchalski, S. Lifson, and H. Eisenberg, J. Polym. Sci., 7, 571 (1951); Katchalski et al., J. Polym. Sci., 8, 476 (1952). (1968).

8. A. J. Shor, K. A. Kraus, W. T. Smith, Jr., and J. S. Johnson, Jr., J. Phys. Chem., 72, 2200

9. P. M. van der Velden, M. H. V. Mulder, L. van der Does, and C. A. Smolders, Polym. Lett. (in press). 
10. H. Yasuda and C. E. Lamaze, Office of Saline Water R \& D Report No. 473, U. S. Dept. of the Interior, 1969.

11. H. Yasuda and C. E. Lamaze, in Permselective Membranes C. E. Rogers, Ed., Marcel Dekker, New York, 1971, pp. 111-134.

12. A. Peterlin, H. Yasuda, and H. G. Olf, J. Appl. Polym. Sci., 16, 865 (1972).

Received April 16, 1975

Revised June 10, 1975 